\title{
Tamoxifen inhibits the proliferation of non-melanoma skin cancer cells by increasing intracellular calcium concentration
}

\author{
GO HASEGAWA ${ }^{1}$, KOTOMI AKATSUKA ${ }^{2}$, YUICHI NAKASHIMA ${ }^{2}$, \\ YUMIKO YOKOE $^{3}$, NARUMI HIGO ${ }^{4}$ and MOTOYUKI SHIMONAKA ${ }^{1}$
}

\author{
${ }^{1}$ Department of Chemistry, Faculty of Science; ${ }^{2}$ Department of Chemistry, Graduate School of Science; \\ ${ }^{3}$ Department of Chemical Sciences and Technology, Graduate School of Chemical Sciences and Technology; \\ ${ }^{4}$ Department of Applied Chemistry, Faculty of Science, Tokyo University of Science, Tokyo 162-8601, Japan
}

Received April 5, 2018; Accepted August 21, 2018

DOI: $10.3892 /$ ijo.2018.4548

\begin{abstract}
Tamoxifen is an estrogen receptor (ER) antagonist used as first-line chemotherapy in breast cancer. Recent studies suggest that tamoxifen may be effective not only for ER-positive but also for ER-negative cancer cases. The aim of the present study was to investigate the antiproliferative effect of tamoxifen against human non-melanoma skin cancer cells. Tamoxifen inhibited the proliferation of the skin squamous cell carcinoma (SCC) cell lines A431, DJM-1 and HSC-1. A431 cells did not express ER- $\alpha$ or $-\beta$, suggesting that tamoxifen may exert antiproliferative effects on skin SCC cells via a non-ER-mediated pathway. Tamoxifen increased the intracellular calcium concentration of skin SCC cells, and this increase in intracellular calcium concentration by calcium ionophore A23187 suppressed the proliferation of skin SCC cells. These data indicate that tamoxifen inhibited the proliferation of human skin SCC cells via increasing intracellular calcium concentration. Voltage-gated calcium channels and non-selective cation channels are involved in the increase in intracellular calcium concentration induced by tamoxifen. The broad-spectrum protein kinase C (PKC) inhibitor phloretin significantly attenuated the antiproliferative effect of tamoxifen on skin SCC cells. From these data, it may be concluded that tamoxifen inhibits the proliferation of skin SCC cells by
\end{abstract}

Correspondence to: Dr Motoyuki Shimonaka, Department of Chemistry, Faculty of Science, Tokyo University of Science, 1-3 Kagurazaka, Shinjuku-ku, Tokyo 162-8601, Japan

E-mail: simonaka@rs.kagu.tus.ac.jp

Abbreviations: ER, estrogen receptor; ERK, extracellular signal-regulated kinase; $\mathrm{GI}_{50}, 50 \%$ growth inhibition; JNK, c-Jun $\mathrm{N}$-terminal kinase; MAPK, mitogen-activated protein kinase; MEK, mitogen-activated protein kinase/extracellular signal-regulated kinase kinase; PKC, protein kinase C; TGF- $\beta$, tumor necrosis factor- $\beta$

Key words: tamoxifen, growth inhibition, calcium, estrogen receptor, calcium channel, cation channel, protein kinase $\mathrm{C}$, mitogenactivated protein kinase induction of extracellular calcium influx via calcium channels in the plasma membrane and by subsequent activation of PKC.

\section{Introduction}

Tamoxifen is an estrogen receptor (ER) antagonist used in the treatment of ER-positive breast cancer $(1,2)$. It is composed of triphenylethylene with an alkylamine side chain, and cytochrome P450 converts tamoxifen to its active form, 4-hydroxytamoxifen (3), which strongly binds to ER- $\alpha$ to antagonize the action of estrogen and exert antitumor effects (4).

The clinical effectiveness of tamoxifen treatment in ER-positive breast cancer patients is well-established $(5,6)$, while some clinical studies demonstrated that tamoxifen administration may also be effective in ER-negative cancer patients (7). The rates of tamoxifen responders with complete/partial response or stable disease among patients with ER-positive and -negative advanced breast cancer were 62 and 27\%, respectively (7). Tamoxifen was also found to be effective for patients with pancreatic cancer $(8,9)$ or recurrent malignant glioma (10). A number of preclinical studies indicated that tamoxifen exerted antitumor effects on ER-negative cancer cells. Tamoxifen inhibited cholangiocarcinoma growth accompanied by suppression of Akt phosphorylation in a mouse xenograft model, and induced apoptosis via activating caspase-3, -8 and -9 in vitro (11). Tamoxifen also effectively inhibited invasion and growth of follicular thyroid cancer cells in vivo and in vitro (12). The combination of tamoxifen and epigallocatechin gallate exerted synergic antiproliferative effects against ER-negative breast cancer xenografts, and inhibited the expression of epidermal growth factor receptor, Akt, mitogen-activated protein kinase (MAPK)/extracellular signal-regulated kinase kinase (MEK) and nuclear factor (NF)- $\mathrm{kB}$ in the xenografts (13). Tamoxifen administration with cell division cycle 42 (Cdc42) inhibitor ML141 treatment suppressed ER-negative breast cancer growth in vivo (14). In vitro studies demonstrated that tamoxifen decreased glioma (15), leukemia (16), hepatocellular carcinoma (17) and prostate cancer (18) cell viability.

The abovementioned evidence indicates that tamoxifen is effective against not only ER-positive but also ER-negative 
cancer cells, although the mechanisms underlying the antitumor effect of tamoxifen against ER-positive and -negative cancer cells are quite different. Although tamoxifen is expected to be effective against various ER-negative cancers, its effect on non-melanoma skin cancer cells, such as squamous cell carcinoma (SCC) cells, remains unknown.

The aim of the present study was to elucidate the antiproliferative effect of tamoxifen on human skin SCC cells and investigate the involvement of intracellular calcium levels in this effect.

\section{Materials and methods}

Materials. Tamoxifen, verapamil, nifedipine, MEK inhibitor PD98059 and calcium ionophore A23187 were purchased from Cayman Chemical, Co. (Ann Arbor, MI, USA). Phloretin was purchased from Calbiochem, Inc. (San Diego, CA, USA). Cell Counting Kit-8 and Fluo 4-AM was purchased from Dojindo Laboratories Co., Ltd. (Kumamoto, Japan). Flufenamic acid was purchased from Santa Cruz Biotechnology, Inc. (Dallas, TX, USA).

Cell culture. The human skin SCC cells A431 and DJM-1, and human acute T-cell leukemia Jurkat cells were obtained from RIKEN BioResource Center (Tsukuba, Japan). Human skin SCC HSC-1 cells, human mammary carcinoma MCF-7 cells and human endometrial adenocarcinoma SNG-II cells were obtained from the Japanese Collection of Research Bioresources Cell Bank (Osaka, Japan). A431 and HSC-1 cells were cultured in Dulbecco's modified Eagle's medium. Jurkat and MCF-7 cells were cultured in RPMI-1640 medium. DJM-1 and SNG-II cells were cultured with Eagle's minimal essential medium and Ham's F12 medium, respectively. All media were supplemented with antibiotics-antimycotics, gentamicin (10 $\mu \mathrm{g} / \mathrm{ml}$, Thermo Fisher Scientific, Inc., Waltham, MA, USA) and $10 \%$ heat-inactivated fetal bovine serum. The cells were maintained at $37^{\circ} \mathrm{C}$ in a humidified atmosphere of $95 \%$ air and $5 \% \mathrm{CO}_{2}$.

Cell proliferation assay. A431, DJM-1 and HSC-1 cells were seeded in 96-well plates (1,000 cells/well) and incubated for $24 \mathrm{~h}$ at $37^{\circ} \mathrm{C}$. The cells were then incubated with tamoxifen (2.5-50 $\mu \mathrm{M})$ for $48 \mathrm{~h}$, and the cell numbers were calculated using the WST- 8 reagent (Cell Counting Kit-8, Dojindo Laboratories).

Reverse transcription-polymerase chain reaction (RT-PCR) analysis. Total RNA was extracted from A431, DJM-1, HSC-1, MCF-7, Jurkat and SNG-II cells with the RNeasy Mini kit (Qiagen GmbH, Hilden, Germany). The Superscript III First-Strand Synthesis System (Thermo Fisher Scientific, Inc.) was then applied. Up to the synthesis of cDNAs, all experiments were conducted under RNase-free conditions. First-strand cDNAs were then used as templates in PCR. The oligonucleotide primers synthesized were 5'-CAGAGAGATGA CTTGGAAGG-3' and 5'-GACTTCAAGGTGCTGGATAG-3' for human ER- $\alpha$; and 5'-TCTACAGTCCTGCTGTGATG-3' and 5'-GTGTACCTGCTCGCTAGAAC-3' for human ER- $\beta$. The experimental conditions for PCR were as follows: $94^{\circ} \mathrm{C}$ for $5 \mathrm{~min}(1 \mathrm{cycle}), 94^{\circ} \mathrm{C}$ for $30 \mathrm{sec}, 60^{\circ} \mathrm{C}$ for $30 \mathrm{sec}, 72^{\circ} \mathrm{C}$ for
$1 \mathrm{~min}$ (30 cycles), $72^{\circ} \mathrm{C}$ for $7 \mathrm{~min}$ ( 1 cycle) for human ER- $\alpha$; and $94^{\circ} \mathrm{C}$ for $5 \mathrm{~min}(1 \mathrm{cycle}), 94^{\circ} \mathrm{C}$ for $30 \mathrm{sec}, 60^{\circ} \mathrm{C}$ for $30 \mathrm{sec}$, $72^{\circ} \mathrm{C}$ for $1 \mathrm{~min}\left(35\right.$ cycles), $72^{\circ} \mathrm{C}$ for $7 \mathrm{~min}$ ( 1 cycle) for human ER- $\beta$. The PCR products were electrophoresed on $1.5 \%$ agarose gel and stained with GelRed (Biotium, Inc., Fremont, CA, USA).

Measurement of intracellular calcium concentration. A431, DJM-1 and HSC-1 cells were seeded in 96-well plates (40,000 cells/well) and incubated for $24 \mathrm{~h}$ at $37^{\circ} \mathrm{C}$. The cells were then incubated with Fluo 4 -AM $(5 \mu \mathrm{M})$ for $1 \mathrm{~h}$ at $37^{\circ} \mathrm{C}$. After residual Fluo 4 removal, tamoxifen (10-50 $\mu \mathrm{M})$ was added to the cells, and the fluorescence emission was measured at $530 \mathrm{~nm}$ in response to excitation at $485 \mathrm{~nm}\left({ }_{485} F^{530}\right)$ by a fluorescence plate reader (CytoFluor Multi-Well Plate Reader Series 4000; PerSeptive Biosystems, Framingham, MA, USA). Intracellular calcium concentration was calculated using the equation of Grynkiewicz et al (19), where $K_{\mathrm{d}}=345 \mathrm{nM}$.

Flow cytometry. A431, DJM-1 and HSC-1 cells were seeded in 6 -well plates $\left(1.2 \times 10^{6}\right.$ cells/well) and incubated for $24 \mathrm{~h}$ at $37^{\circ} \mathrm{C}$. The cells were then incubated with Fluo 4-AM $(5 \mu \mathrm{M})$ for $1 \mathrm{~h}$ at $37^{\circ} \mathrm{C}$. After residual Fluo 4 removal, tamoxifen $(100 \mu \mathrm{M})$ was added to the cells, and fluorescence was measured by FACSCanto II flow cytometer (BD Biosciences, San Jose, CA, USA).

Western blot analysis. A431, DJM-1 and HSC-1 cells were seeded in $60-\mathrm{mm}$ dishes $\left(3 \times 10^{6}\right.$ cells/dish) and incubated for $24 \mathrm{~h}$ at $37^{\circ} \mathrm{C}$. The cells were then incubated with tamoxifen (20 or $30 \mu \mathrm{M}$ ) for $0,10,20,30$ or $60 \mathrm{~min}$. After extensive washing with phosphate-buffered saline, the cells were lysed with cell lysis buffer $(0.01 \mathrm{M}$ phosphate, $0.15 \mathrm{M} \mathrm{NaCl}, 5 \mathrm{mM}$ EDTA, $1 \%$ Nonidet P-40, $0.1 \%$ sodium dodecyl sulfate and $0.1 \%$ sodium deoxycholate). The protein concentration of the cell lysates was measured with the Protein Assay Bicinchoninate Kit (Nacalai Tesque, Inc., Kyoto, Japan).

Cell lysates $(90 \mu \mathrm{g})$ were electrophoresed on a polyacrylamide gel under non-reducing conditions, and resolved proteins were electrophoretically transferred to a PVDF membrane. The membrane was incubated for $1 \mathrm{~h}$ in a blocking solution (5\% skimmed milk in TBS containing $0.05 \%$ Tween-20) and probed with anti-human phospho-protein kinase $\mathrm{C}$ (PKC) (pan) rabbit polyclonal antibody (1:2,000 diluted in TBS containing $1 \%$ skimmed milk and $0.05 \%$ Tween-20; Cell Signaling Technology, Inc., Danvers, MA, USA), antihuman PKC $\alpha$ rabbit polyclonal antibody $(1: 2,000$, Cell Signaling Technology), anti-human PKCס rabbit monoclonal antibody (D10E2, 1:2,000, Cell Signaling Technology), antihuman phospho-p44/42 MAPK rabbit monoclonal antibody (D13.14.4E, 1:2,000, Cell Signaling Technology), anti-human p44/42 MAPK rabbit monoclonal antibody $(1: 2,000$, Cell Signaling Technology) or anti-human glyceraldehyde3-phosphate dehydrogenase (GAPDH) rabbit monoclonal antibody (14C10, 1:1,000, Cell Signaling Technology) overnight at $4^{\circ} \mathrm{C}$. After extensive washing with TBS containing $0.05 \%$ Tween-20, the membrane was further incubated with a horseradish peroxidase-conjugated goat anti-rabbit IgG antibody (1:4,000 dilution; Cell Signaling Technology), and the bands of antigens were detected by chemiluminescence of the 

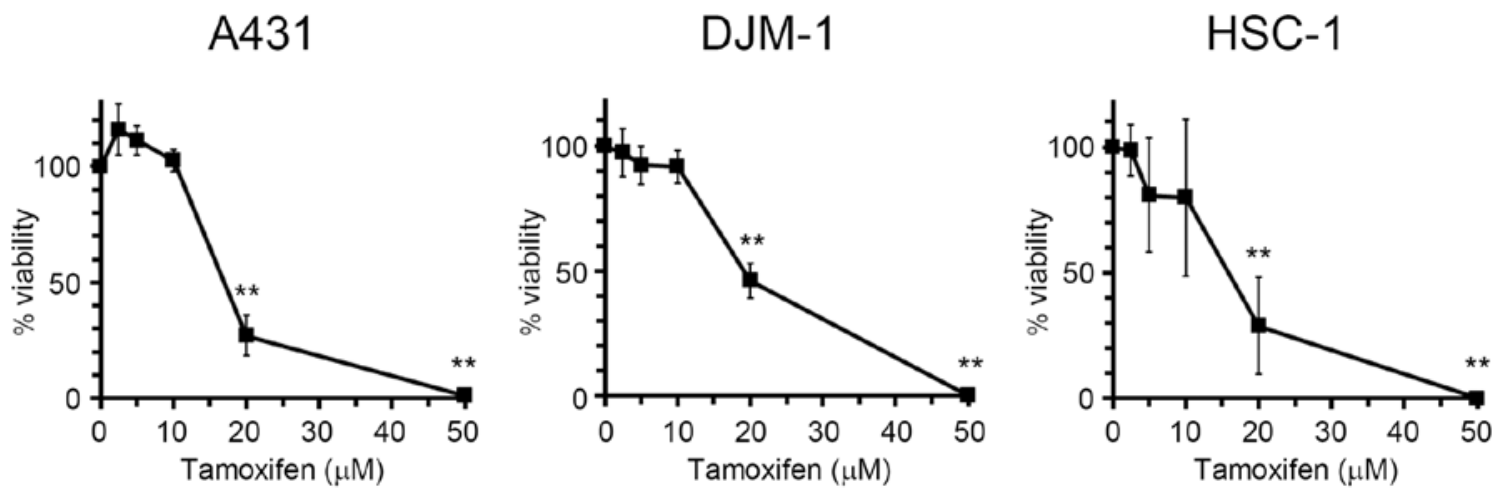

Figure 1. Antiproliferative effects of tamoxifen on skin SCC cells. A431, DJM-1 and HSC-1 cells were incubated with tamoxifen for 48 h, and the cell numbers were calculated by the WST assay. The number of cells in the control group (tamoxifen $0 \mu \mathrm{M}$ ) was set as $100 \%$ and the cell counts for all treatment groups are expressed as a percentage against the control. ${ }^{* *} \mathrm{P}<0.01$ (vs. tamoxifen $0 \mu \mathrm{M}$, Dunnett's test). SCC, squamous cell carcinoma.

product of peroxidase reaction using Luminata Forte Western HRP Substrate (EMD Millipore Corp., Darmstadt, Germany).

Data analysis. All data are expressed as mean \pm standard deviation. The significance of the difference between two groups was analyzed by Student's t-tests and among more than three groups by one-way and two-way analysis of variance followed by multiple comparison tests, Dunnett's test or Tukey's test. A P-value of $<0.05$ was considered to indicate a statistically significant difference. Data analysis was performed using JMP 9 software (SAS Institute, Inc., Cary, NC, USA).

\section{Results}

Tamoxifen exerts antiproliferative effects on human skin $S C C$ cells. Tamoxifen is known to inhibit cell proliferation in several types of cancer, such as breast cancer (20). In order to evaluate the antiproliferative effect of tamoxifen on human skin SCC cells, A431, DJM-1 and HSC-1 cells were incubated with tamoxifen, and the cell numbers were then estimated by the WST assay (Fig. 1). Tamoxifen inhibited the proliferation of skin SCC cells dose-dependently at concentrations of 2.5-50 $\mu \mathrm{M}$. The $\mathrm{GI}_{50}$ (50\% growth inhibition) values of tamoxifen for A431, DJM-1 and HSC-1 cells were 17.6, 19.4 and $15.6 \mu \mathrm{M}$, respectively.

Tamoxifen has been shown to antagonizeER function, which is a well-known mechanism mediating the antiproliferative effects of tamoxifen against ER-positive cancer cells. Thus, the expression of ERs in skin SCC cells was investigated by RT-PCR (Fig. 2). A431 cells did not express either ER- $\alpha$ or $-\beta$, DJM- 1 cells expressed both ER- $\alpha$ and $-\beta$, whereas HSC- 1 cells slightly expressed ER- $\alpha$ and $-\beta$. It has been reported that tamoxifen may inhibit the proliferation of both ER-positive and -negative cells (21). These results indicate that tamoxifen may exert its antiproliferative effect on certain types of skin SCC cells via a non-ER-mediated pathway.

Tamoxifen increases intracellular calcium concentration in skin SCC cells. It was reported that tamoxifen-induced apoptosis is mediated by intracellular calcium signals in human hepatoblastoma HepG2 cells (17). To elucidate the mechanism underlying the tamoxifen-induced antiproliferative effect on

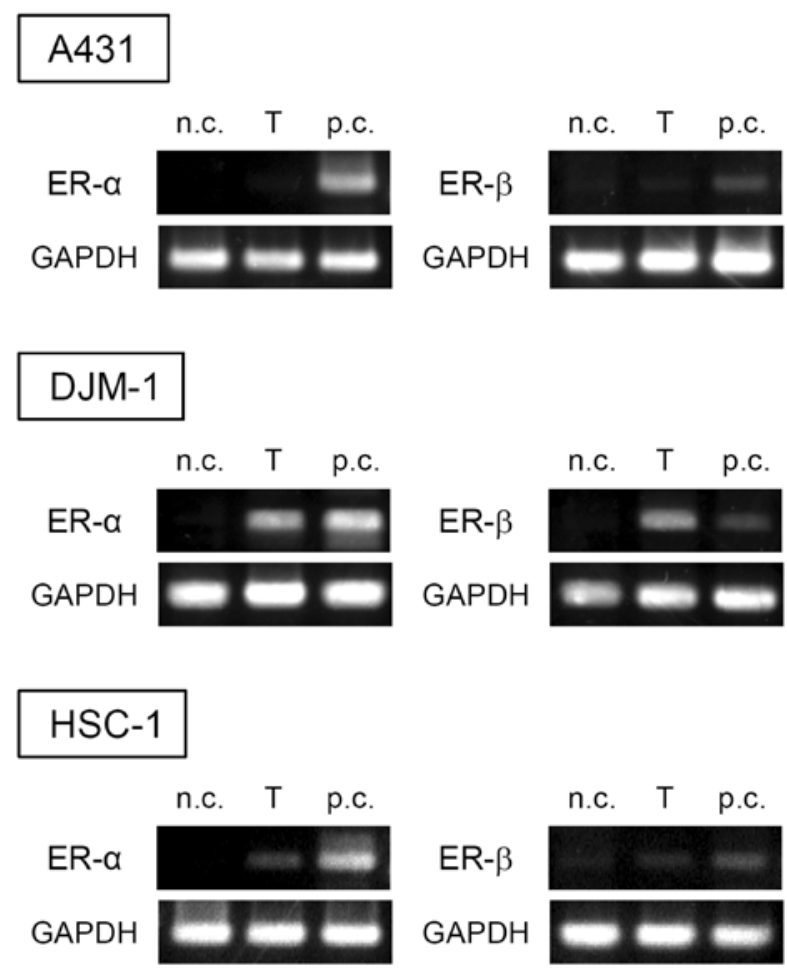

Figure 2. Expression of ER- $\alpha$ and $-\beta$ in skin SCC cells. The expression of ER- $\alpha$ and $-\beta$ in A431, DJM- 1 and HSC- 1 cells was evaluated by RT-PCR. The data shown are from one experiment representative of at least three separate experiments. n.c., negative control (Jurkat for ER- $\alpha$, SNG-II for ER- $\beta$ ); T, target cell (A431, DJM-1 or HSC-1), p.c., positive control (MCF-7). ER, estrogen receptor; SCC, squamous cell carcinoma; RT-PCR, reverse transcription-polymerase chain reaction.

skin SCC cells, the changes in intracellular calcium concentration were investigated. As shown in Fig. 3A, tamoxifen markedly increased intracellular calcium concentration in A431, DJM-1 and HSC-1 cells in a dose-dependent manner. The increase in intracellular calcium levels in A431, DJM-1 and HSC-1 cells induced by tamoxifen was also evaluated by flow cytometry (Fig. 3B). The data suggest that tamoxifen induces calcium influx in skin SCC cells.

Increase in intracellular calcium concentration suppresses the proliferation of skin SCC cells. In order to confirm the 


\section{A}

A431

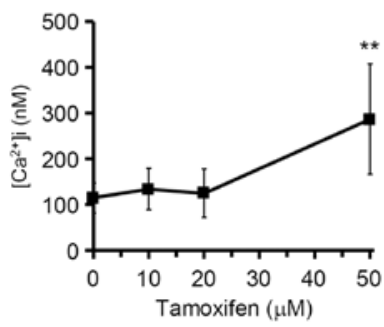

B

A431

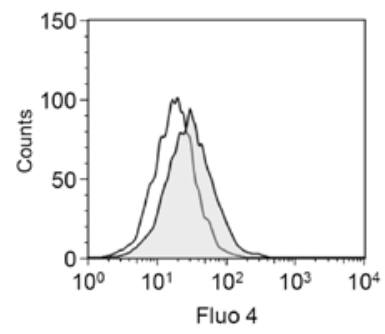

DJM-1

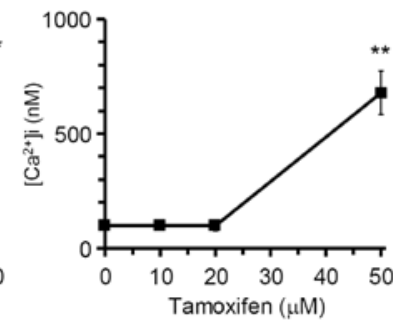

DJM-1

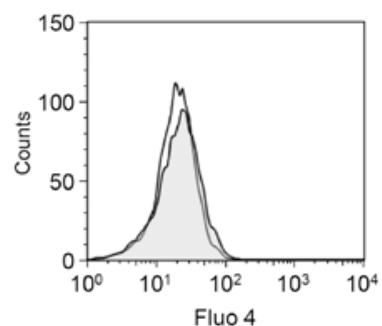

HSC-1

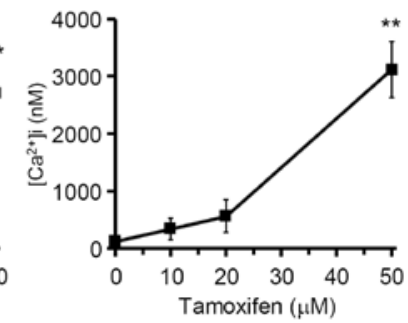

HSC-1

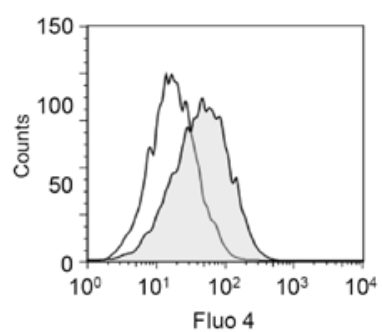

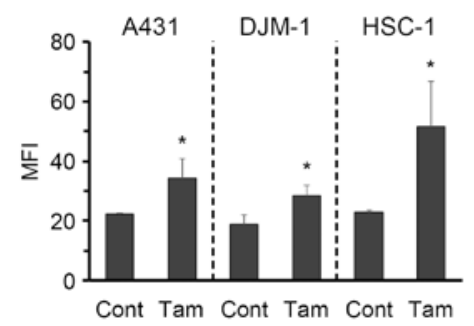

Figure 3. Tamoxifen increased intracellular calcium concentration of skin SCC cells. (A) A431, DJM-1 and HSC-1 cells were incubated with Fluo 4-AM (5 $\mu$ M) for $1 \mathrm{~h}$, and then tamoxifen was added at various concentrations. Fluorescence derived from Fluo 4 was monitored by a fluorescence plate reader, and intracellular calcium concentration was calculated. ${ }^{* *} \mathrm{P}<0.01$ (vs. tamoxifen $0 \mu \mathrm{M}$, Dunnett's test). (B) The increase of intracellular calcium concentration induced by tamoxifen (100 $\mu \mathrm{M}$, shaded area) in A431, DJM-1 and HSC-1 cells was also evaluated by FACS. Open areas represent control. The data shown are from one experiment representative of at least three separate experiments. The mean fluorescence intensity (MFI) data generated by the results of flow cytometry are also shown. Cont, control; Tam, tamoxifen $(100 \mu \mathrm{M})$. "P<0.05 (vs. Cont, Student's t-test). SCC, squamous cell carcinoma.

association between the increase in intracellular calcium concentration and growth inhibition of skin SCC cells, A431, DJM-1 and HSC-1 cells were incubated with calcium ionophore A23187 at various concentrations $(0.5-5 \mu \mathrm{M})$, and the cell numbers were then calculated (Fig. 4A). A23187 significantly inhibited the growth of skin SCC cells, indicating that the increase in intracellular calcium concentration caused growth inhibition of skin SCC cells. It was confirmed that A23187 $(0.5-5 \mu \mathrm{M})$ increased the intracellular calcium levels in A431, DJM-1 and HSC-1 cells (Fig. 4B). Following addition of A23187 at $>2 \mu \mathrm{M}$ for A431 cells and $>1 \mu \mathrm{M}$ for HSC- 1 cells, intracellular calcium concentration could not be measured, due to the excess fluorescence over the detection limits. These results indicate that tamoxifen inhibited the proliferation of human skin SCC cells via increasing intracellular calcium concentration.

Voltage-gated calcium channels as well as non-selective cation channels are involved in the increase in intracellular calcium concentration in skin SCC cells induced by tamoxifen. Two major pathways are involved in the increase in intracellular cytosolic calcium concentration: Release from endoplasmic reticulum calcium stores to the cytosol, and extracellular calcium influx via plasma membrane calcium channels (22). To elucidate which pathway is implicated in the increase in intracellular cytosolic calcium concentration in skin SCC cells induced by tamoxifen, it was first investigated whether tamoxifen induced calcium release from intracellular stores. A431, DJM-1 and HSC-1 cells were incubated with Fluo 4-AM in HEPES buffer solution without calcium, followed by the addition of tamoxifen. The fluorescence derived from Fluo 4 was monitored and intracellular calcium concentration was calculated. As shown in Fig. 5, addition of tamoxifen did not increase the intracellular cytosolic calcium concentration in skin SCC cells when using calcium-depleted buffer solution. However, thapsigargin (23), which discharged intracellular calcium stores by potently inhibiting endoplasmic reticulum calcium ATPase, increased the intracellular cytosolic calcium concentration in skin SCC cells. Addition of thapsigargin increased the intracellular calcium concentration in A431, DJM-1 and HSC-1 cells up to $231.8 \pm 57.8$, $299.2 \pm 124.9$ and $438.1 \pm 252.4 \mu \mathrm{M}$, respectively, whereas the 
A

A431

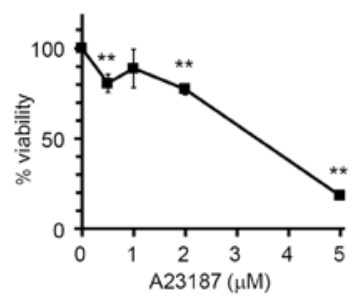

B

A431

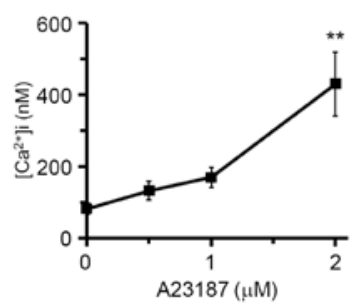

DJM-1

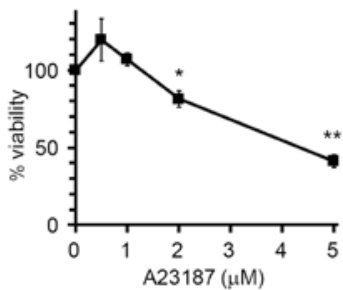

HSC-1

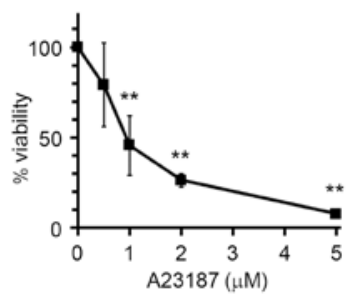

DJM-1

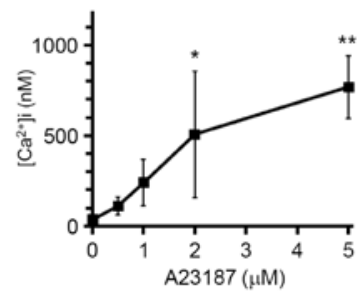

HSC-1

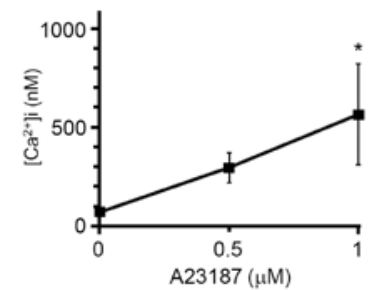

Figure 4. Increase of intracellular calcium concentration inhibited the proliferation of skin SCC cells. (A) A431, DJM-1 and HSC-1 cells were incubated with calcium ionophore A23187 at various concentrations for $48 \mathrm{~h}$, and the cell numbers were calculated by the WST assay. The number of cells in the control group (A23187 $0 \mu \mathrm{M}$ ) was set as 100\% and the cell counts for all treatment groups are expressed as a percentage against the control. (B) A431, DJM-1 and HSC-1 cells were incubated with Fluo 4-AM $(5 \mu \mathrm{M})$ for $1 \mathrm{~h}$, followed by the addition of various concentrations of A23187. Fluorescence derived from Fluo 4 was monitored by a fluorescence plate reader and the intracellular calcium concentration was calculated. ${ }^{*} \mathrm{P}<0.05$ and ${ }^{* *} \mathrm{P}<0.01$ (vs. A23187 $0 \mu \mathrm{M}, \mathrm{Dunnett}$ 's test). Data are expressed as mean \pm standard deviation calculated from the result of at least three separate experiments. SCC, squamous cell carcinoma.

\section{A431}

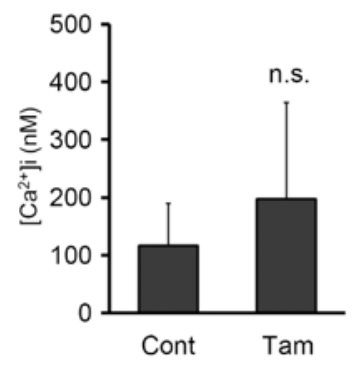

DJM-1

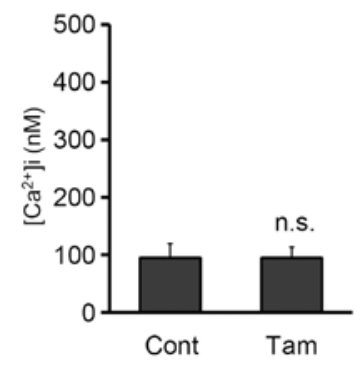

HSC-1

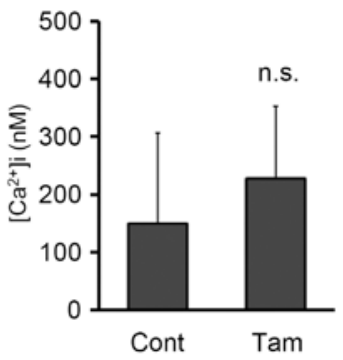

Figure 5. Tamoxifen did not induce calcium release from ER in skin SCC cells. A431, DJM-1 and HSC-1 cells were incubated with Fluo 4-AM (5 $\mu$ M) in recording buffer without calcium for $1 \mathrm{~h}$, and then tamoxifen (50 $\mu \mathrm{M}$, Tam) or DMSO (Cont) was added. Fluorescence derived from Fluo 4 was monitored, and intracellular calcium concentration was calculated as described in 'Materials and methods'. Cont, control; Tam, tamoxifen; n.s., not significant (vs. Cont, Student's t-test). SCC, squamous cell carcinoma.

intracellular calcium concentrations in A431, DJM-1 and HSC-1 cells without thapsigargin were $116.0 \pm 73.9,95.2 \pm 24.4$ and $149.3 \pm 157.3 \mu \mathrm{M}$, respectively. These data suggest that the tamoxifen-induced increase in intracellular cytosolic calcium concentration was not due to calcium release from the endoplasmic reticulum stores.

It was next investigated whether extracellular calcium influx via plasma membrane calcium channels was involved in the increase in intracellular calcium concentration in skin SCCs induced by tamoxifen. The tamoxifen-induced increase in intracellular cytosolic calcium concentration was significantly inhibited by nifedipine, a voltage-gated calcium channel inhibitor, and flufenamic acid, a non-selective cation channel blocker (Fig. 6A). Verapamil, another voltage-gated calcium channel inhibitor, significantly inhibited the increase in intracellular cytosolic calcium concentration in DJM-1 cells. From these data, both voltage-gated calcium channels and non-selective cation channels appear to be involved in the increase in intracellular cytosolic calcium concentration in skin SCC cells induced by tamoxifen. As shown in Fig. 6B, blockage of calcium influx by voltage-gated calcium channel and non-selective cation channel inhibitors attenuated the tamoxifen-induced toxicity on skin SCC cells, indicating that tamoxifen-induced cytotoxicity is mediated by calcium influx.

$P K C$ is involved in the antiproliferative effect of tamoxifen on skin SCC cells. The results in Fig. 3 indicate that tamoxifen increased the intracellular cytosolic calcium concentration of skin SCC cells. Calcium is a well-known second messenger that is involved in a number of intracellular signal transduction pathways, such as the PKC pathway (24). In order to confirm the involvement of PKC in tamoxifen-induced growth inhibition of 


\section{A}

A431

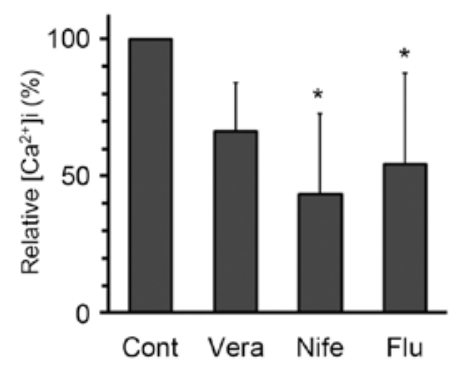

B

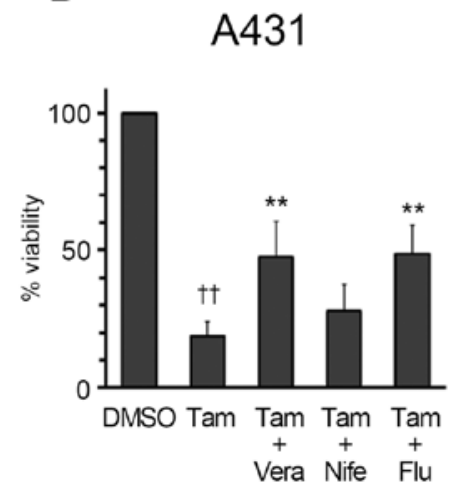

DJM-1

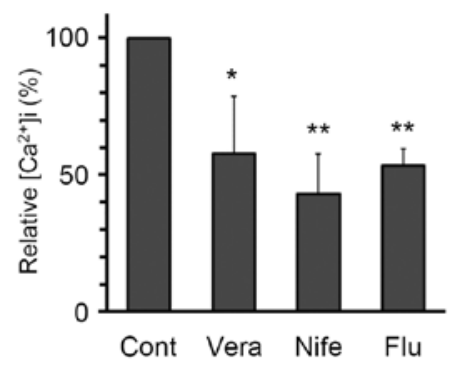

DJM-1

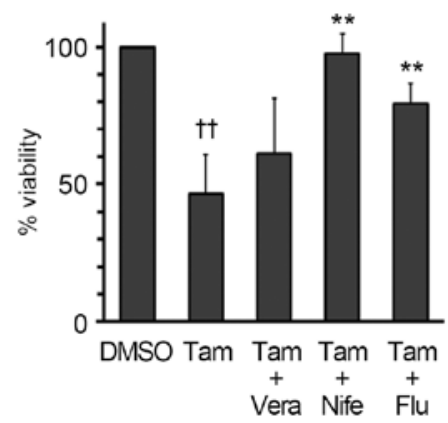

HSC-1

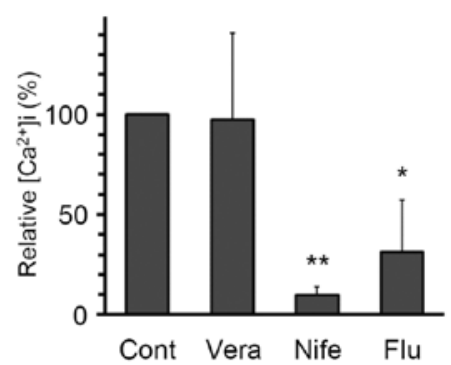

HSC-1

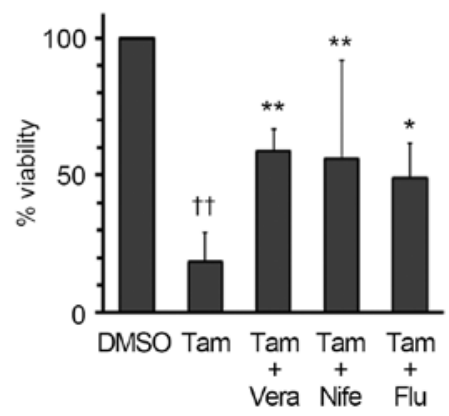

Figure 6. Inhibitory effects of calcium channel blockers on tamoxifen-induced calcium influx in skin SCC cells. (A) A431, DJM-1 and HSC-1 cells were pre-incubated with verapamil (100 $\mu \mathrm{M}$, Vera), nifedipine (100 $\mu \mathrm{M}$, Nife), flufenamic acid (100 $\mu \mathrm{M}$, Flu) or DMSO (Cont) for $1 \mathrm{~h}$, and then incubated with Fluo 4-AM $(5 \mu \mathrm{M})$ for $1 \mathrm{~h}$. Calcium influx derived from the addition of $50 \mu \mathrm{M}$ tamoxifen (Tam) was monitored, and the relative intracellular calcium concentration was calculated. ${ }^{*} \mathrm{P}<0.05,{ }^{* *} \mathrm{P}<0.01$ (vs. Cont, Dunnett's test). (B) A431, DJM-1 and HSC-1 cells were pre-incubated with $100 \mu \mathrm{M}$ Vera, $100 \mu \mathrm{M}$ Nife, $100 \mu \mathrm{M}$ Flu or dimethyl sulfoxide (DMSO) for $1 \mathrm{~h}$, and then incubated with Tam ( $20 \mu \mathrm{M}$ for A431 and DJM-1 cells, $25 \mu \mathrm{M}$ for HSC-1 cells) for $48 \mathrm{~h}$, and the cell numbers were calculated by the WST assay. The number of cells in the control group (DMSO) was set as $100 \%$ and the cell counts for all treatment groups are expressed as a percentage against the control. ${ }^{\dagger} \mathrm{P}<0.01$ (vs. DMSO), ${ }^{*} \mathrm{P}<0.05,{ }^{* *} \mathrm{P}<0.01$ (vs. Tam, Tukey's test). SCC, squamous cell carcinoma.

skin SCC cells, A431, DJM-1 and HSC-1 cells were incubated with tamoxifen in the presence of the broad-spectrum PKC inhibitor phloretin, and the cell numbers were then calculated by the WST assay (Fig. 7A). Tamoxifen-induced growth inhibition of skin SCC cells was markedly recovered by phloretin. The inhibition ratios of phloretin against tamoxifen-induced growth inhibition were 33.7, 24.0 and 44.2\% for A431, DJM-1 and HSC-1 cells, respectively. These data demonstrated that $\mathrm{PKC}$ is involved in the antiproliferative effect of tamoxifen on skin SCC cells.

Calcium is also involved in the MAPK pathway (25); thus, the involvement of MAPK/MEK in tamoxifen-induced growth inhibition of skin SCC cells was investigated. A431, DJM-1 and HSC-1 cells were incubated with tamoxifen in the presence of the selective MEK inhibitor PD98059, and the cell numbers were then calculated by the WST assay (Fig. 7A). Although the effect was not statistically significant, PD98059 tended to recover the viability of skin SCC cells against tamoxifeninduced growth inhibition. The inhibition ratios of PD98059 against tamoxifen-induced growth inhibition were 23.0, 15.5 and $16.5 \%$ for A431, DJM-1 and HSC-1 cells, respectively. The results from western blot analysis demonstrated that tamoxifen induced the phosphorylation of PKC and extracellular signalregulated kinase1/2 (ERK1/2) in A431, DJM-1 and HSC-1 cells (Fig. 7B). Taken together, these data indicate that the PKC and MAPK pathways may be involved in the antiproliferative effects of tamoxifen on skin SCC cells; the contribution of the
MAPK pathway, however, is smaller compared with that of the PKC pathway.

\section{Discussion}

One of the major functions of tamoxifen is to inhibit the proliferation of cancer cells by antagonizing ER-induced signaling pathways. The effectiveness of tamoxifen against several types of ER-negative cancer cells (e.g., glioma and hepatocellular carcinoma) is also widely reported (26); however, the effect of tamoxifen on non-melanoma skin cancer remained unknown. In the present study, it was demonstrated that tamoxifen inhibited the proliferation of human skin SCC cells via a non-ER-mediated pathway. Tamoxifen increased the intracellular calcium concentration in skin SCC cells by influx of extracellular calcium through voltage-gated calcium channels and non-selective cation channels, resulting in the activation of PKC.

Tamoxifen inhibited the proliferation of A431, DJM-1 and HSC-1 cells dose-dependently at concentrations of $2.5-50 \mu \mathrm{M}$ (Fig. 1). The $\mathrm{GI}_{50}$ values for A431, DJM-1 and HSC-1 cells were 17.6, 19.4 and $15.6 \mu \mathrm{M}$, respectively. ER-positive cancer cells are usually more sensitive to tamoxifen compared with ER-negative cells. For example, the $\mathrm{GI}_{50}$ values for the ER-positive breast cancer cell lines MCF-7 and T47D were reported to be $<3 \mu \mathrm{M}$, whereas the $\mathrm{GI}_{50}$ values for the ER-negative breast cancer cell lines MDA-MB-231 and 
A
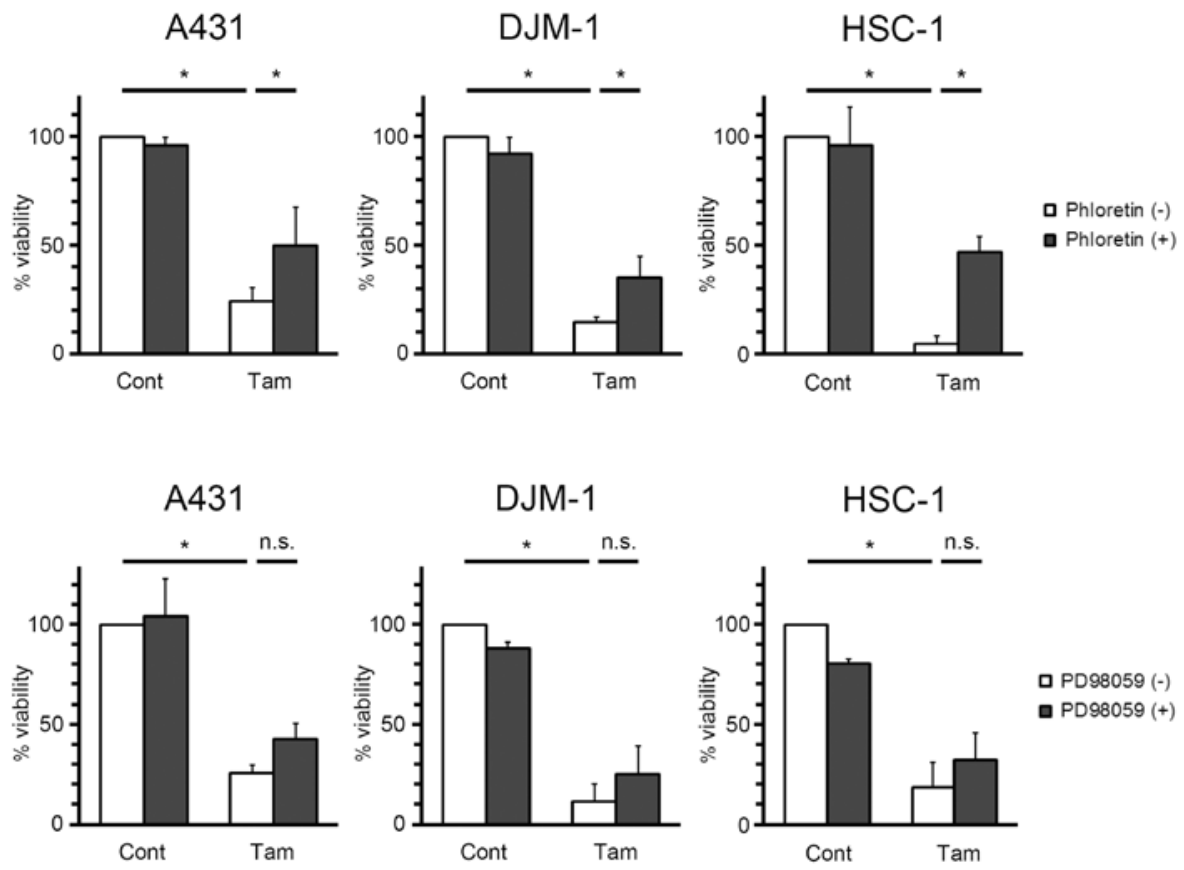

口 PD98059 (-)

口 PD98059 (+)

B

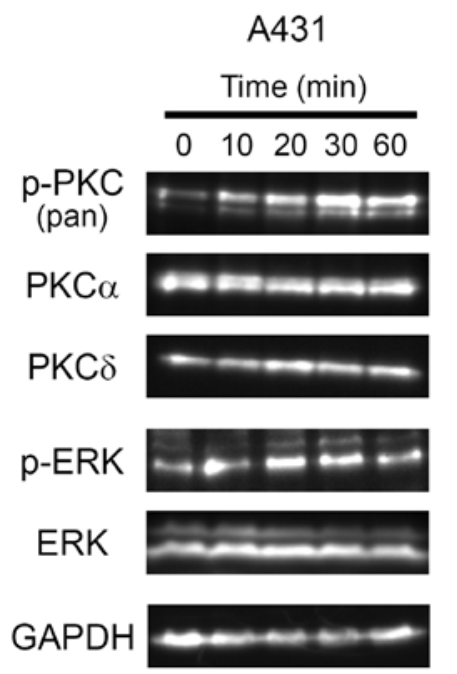

DJM-1

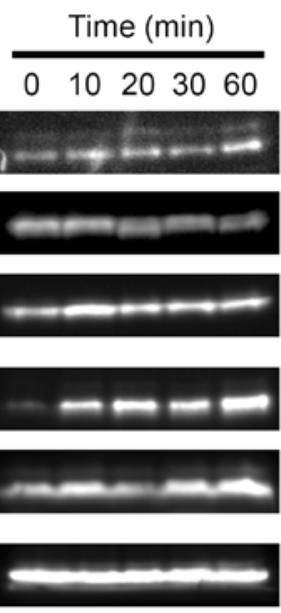

HSC-1

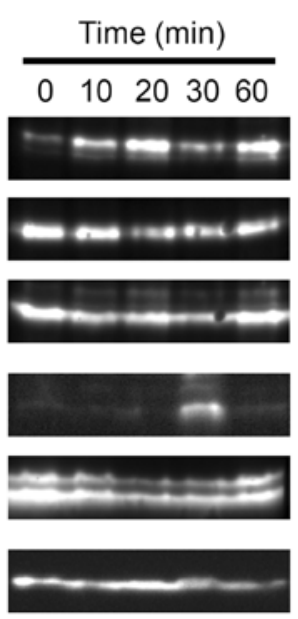

Figure 7. Effects of PKC and MEK inhibitors on the antiproliferative activity of tamoxifen on skin SCC cells. (A) A431, DJM-1 and HSC-1 cells were preincubated with (+) or without (-) phloretin $(500 \mu \mathrm{M}$, upper row) or PD98059 $(25 \mu \mathrm{M}$, lower row) for $1 \mathrm{~h}$, and then incubated with tamoxifen (Tam; $20 \mu \mathrm{M}$ for A431 and HSC-1 cells, $30 \mu \mathrm{M}$ for DJM-1 cells) for $48 \mathrm{~h}$. The cell numbers were calculated by the WST assay. The number of cells in the control group (Cont) was set as $100 \%$ and the cell counts for all treatment groups are expressed as a percentage against the control. "P<0.05 (Tukey's test), n.s., not significant. (B) A431, DJM-1 and HSC-1 cells were incubated with Tam ( $20 \mu \mathrm{M}$ for A431 and HSC-1 cells, $30 \mu \mathrm{M}$ for DJM-1 cells) for various times, and the expression of phospho-PKC [p-PKC (pan)], PKC $\alpha$, PKC $\delta$, phospho-ERK (p-ERK), ERK and GAPDH were evaluated by western blotting. The data shown are from one experiment representative of at least three separate experiments. PKC, protein kinase C; ERK, extracellular signal-regulated kinase; MEK, mitogen-activated protein kinase/extracellular signal-regulated kinase kinase; SCC, squamous cell carcinoma.

HCC1937 was $>3 \mu \mathrm{M}$ (27). Guo et al (4) reported that the mean value of $\mathrm{GI}_{50}$ of tamoxifen over all 39 cancer cell lines (MG-MID), including both ER-positive and -negative cell lines, was $7.41 \mu \mathrm{M}$. Although the $\mathrm{GI}_{50}$ values of tamoxifen for A431, DJM-1 and HSC-1 cells were higher compared with the MG-MID of tamoxifen, the antiproliferative effect of tamoxifen on skin SCC cells was notable. A431 cells do not express either ER- $\alpha$ or $-\beta$ (Fig. 2), suggesting that the growth inhibitory effects of tamoxifen on skin SCC cells were mediated via a non-ER pathway.
In order to elucidate the mechanism underlying the antiproliferative effects of tamoxifen on skin SCC cells, the change in intracellular calcium concentration during tamoxifen treatment was investigated. As shown in Fig. 3A, tamoxifen significantly increased intracellular calcium concentration in A431, DJM-1 and HSC-1 cells, thereby inhibiting the growth of skin SCC cells (Fig. 4A). Thus, it was strongly suggested that the tamoxifen-induced growth inhibition of skin SCC cells was caused by an increase in the intracellular calcium concentration. 
Tamoxifen at a dose of $20 \mu \mathrm{M}$ reduced the viability of skin SCC cells to $50-70 \%$ (Fig. 1), although the same concentration of tamoxifen did not cause a marked elevation in intracellular calcium concentration (Fig. 3A). This discrepancy may be explained by the differences in the incubation times of skin SCC cells with tamoxifen in these assays. To evaluate the effect of tamoxifen on cell viability, skin SCC cells were incubated with tamoxifen for $48 \mathrm{~h}$. However, intracellular calcium concentration was measured immediately following the addition of tamoxifen to skin SCC cells, as mentioned in 'Materials and methods'. These differences in incubation times of skin SCC cells with tamoxifen in each assay were the cause of the variations in the effective tamoxifen concentration. Although the addition of $20 \mu \mathrm{M}$ tamoxifen did not immediately induce a marked increase in intracellular calcium concentration, it may have induced a moderate calcium influx, resulting in SCC cell death.

Additionally, these differences in incubation time of skin SCC cells in each assay exclude the possibility of calcium influx through a weakened cell membrane by tamoxifen. As mentioned above, the intracellular calcium concentration was measured immediately following the addition of tamoxifen to skin SCC cells. Thus, it is strongly suggested that the plasma membrane of skin SCC cells was intact when intracellular calcium concentration was measured. Therefore, calcium influx may not be triggered in dying cells when the plasma membrane is likely not intact.

To identify the mechanism underlying the tamoxifeninduced increase of cytosolic calcium concentration in skin SCC cells, it was investigated whether tamoxifen induced calcium release from the endoplasmic reticulum stores. It has been reported that thapsigargin discharged intracellular calcium stores by potently inhibiting endoplasmic reticulum calcium ATPase, followed by an increase of the intracellular cytosolic calcium concentration (23). Although thapsigargin increased intracellular cytosolic calcium concentration in skin SCC cells, tamoxifen did not (Fig. 5), indicating that tamoxifen did not induce the efflux of calcium from endoplasmic reticulum stores to the cytosol in skin SCC cells.

Extrinsic stimuli may cause extracellular calcium influx into the cell via calcium-permeable channels, such as voltagegated calcium channels and non-selective cation channels. The effects of voltage-gated calcium channel inhibitors, such as verapamil and nifedipine, and the non-selective cation channel blocker, flufenamic acid, on tamoxifen-induced calcium influx in skin SCC cells were examined in the present study. Nifedipine and flufenamic acid significantly inhibited tamoxifen-induced calcium influx in A431, DJM-1 and HSC-1 cells (Fig. 6A), indicating that both voltage-gated calcium channels and non-selective cation channels were involved in tamoxifen-induced calcium influx. In our experiments, tamoxifen immediately increased intracellular calcium concentration in skin SCC cells (Fig. 3). Thus, tamoxifen may not act by increasing the expression level of calcium channels on the plasma membrane. These findings suggest that tamoxifen modulates the activity of voltage-gated calcium channels and non-selective cation channels to induce extracellular calcium influx. Kim et al (17) reported that tamoxifen-induced calcium influx in ER-negative human hepatoblastoma HepG2 cells was significantly inhibited by flufenamic acid, although verapamil or nifedipine did not affect this tamoxifen-induced calcium influx. This observation together with our data suggest that the mechanism of tamoxifen-induced calcium influx into the cytosol in ER-negative cancer cells may vary among different cancer types. Zhang et al reported that tamoxifen enhanced and prolonged ATP-induced intracellular calcium elevation in both ER-positive MCF-7 breast cancer cells and ER-negative C6 glioma cells (28). It was also reported that tamoxifen increased intracellular calcium concentration in MG-63 osteosarcoma cells (29), which express both ER- $\alpha$ and $-\beta$ (30). Although an increase of tamoxifen-induced intracellular calcium concentration may be accompanied by cytotoxicity (31), the effect of ER on tamoxifen-induced intracellular calcium increase leading to cell death and the detailed mechanism underlying tamoxifeninduced calcium influx will be investigated in future studies.

Verapamil significantly inhibited the increase in intracellular cytosolic calcium concentration of DJM-1 cells, and tended to inhibit the increase in intracellular cytosolic calcium concentration in A431 cells (Fig. 6A). However, verapamil did not inhibit the increase in intracellular cytosolic calcium concentration in HSC-1 cells, although nifedipine almost completely inhibited the increase in intracellular cytosolic calcium concentration in this cell line. It is reported that the inhibitory mechanisms of action of verapamil and nifedipine on voltage-gated calcium channels are different (32). Phenylalkylamines (e.g., verapamil) bind to the V-binding site in L-type calcium channels, while dihydropyridines (e.g., nifedipine) bind to the N-binding site. Administration of these two types of calcium channel inhibitors results in different antihypertensive effects in patients with essential hypertension and in normal subjects (33). Although the details are unknown, the mechanism of voltage-gated calcium channel activation by tamoxifen may differ in skin SCC cells.

Tamoxifen-induced growth inhibition of skin SCC cells was significantly attenuated by the addition of the broadspectrum PKC inhibitor phloretin (Fig. 7A). This observation suggests that PKC may be involved in the calcium-mediated growth-inhibitory signaling in skin SCC cells induced by tamoxifen. The phosphorylation of PKC $\alpha$ increases during tamoxifen-induced apoptosis in ER-negative human breast cancer MCF-7/ADR cells (21), and our data suggested that some isoforms of PKC in skin SCC cells were phosphorylated during incubation with tamoxifen (Fig. 7B). The role of the PKC isoform involved in tamoxifen-induced growth inhibition of skin SCC cells remains to be elucidated.

There was a tendency for the MEK inhibitor PD98059 to attenuate tamoxifen-induced growth inhibition of skin SCC cells (Fig. 7A), suggesting that the MAPK pathway was also involved in calcium-mediated growth-inhibitory signaling in skin SCC cells induced by tamoxifen. It is well-known that intracellular calcium affects the MAPK pathway via small GTPase Ras activation (34). Tamoxifen increased the phosphorylation of ERK1/2, downstream kinases of MEK, in A431, DJM-1 and HSC-1 cells (Fig. 7B). Xie et al (21) reported that tamoxifen induced ERK1/2 phosphorylation in both ER-positive and -negative breast cancer cells. Therefore, the MEK/ERK pathway may be involved in the tamoxifen-induced growth inhibition of cancer cells, although its contribution is limited.

Our data indicate that tamoxifen activates the PKC and MAPK pathways during growth inhibition in skin SCC cells. However, the downstream effect of PKC and MAPK activation 
on tamoxifen-induced growth inhibition of skin SCC cells remains unclear. It has been reported that PKC and MAPK activation affect the expression and trafficking of tumor necrosis factor (TGF)- $\beta$, which inhibits cancer cell growth. TGF- $\beta$ is known to inhibit the growth of A431 cells (35), and PKC- $\varepsilon$ and $-\zeta$ are involved in the trafficking of TGF- $\beta$ (36). P38 MAPK, ERK and JNK are involved in the expression of TGF- $\beta$ in fibroblasts during apoptosis (37). It was also reported that tamoxifen induces the expression of TGF- $\beta$ in ER-positive and -negative breast cancer cells (38). In addition, it was observed that tamoxifen increased the transcription level of TGF- $\beta$ in HSC-1 cells (data not shown). Taken together, these findings indicate that tamoxifen may induce TGF- $\beta$ expression by activating the PKC and MAPK pathways through an increase in intracellular calcium concentration, resulting in growth inhibition of skin SCC cells.

In conclusion, the data of the present study demonstrated that tamoxifen inhibits the proliferation of skin SCC cells. To the best of our knowledge, this is the first report to elucidate the antiproliferative effect of tamoxifen on skin SCC cells. Tamoxifen induces extracellular calcium influx into the cytoplasm via voltage-gated calcium channels and non-selective cation channels, and then activates the PKC and MEK/ERK pathways. These data suggest that the increase in intracellular calcium concentration is key to the antiproliferative effects of tamoxifen on ER-negative cancer cells.

\section{Acknowledgements}

Not applicable.

\section{Funding}

No funding was received.

\section{Availability of data and materials}

The datasets used and/or analyzed during this study are available from the corresponding author on reasonable request.

\section{Authors' contributions}

GH was a major contributor to designing the study, performing the experiments, analyzing the data and writing the manuscript. KA, YN, YY and NH performed the experiments. MS designed the study and wrote the manuscript. All authors have read and approved the final version of the manuscript.

\section{Ethics approval and consent to participate}

Not applicable.

\section{Patient consent for publication}

Not applicable.

\section{Competing interests}

The authors declare that they have no competing interests to disclose.

\section{References}

1. Osborne CK: Tamoxifen in the treatment of breast cancer. N Engl J Med 339: 1609-1618, 1998.

2. Johnston SR and Dowsett M: Aromatase inhibitors for breast cancer: Lessons from the laboratory. Nat Rev Cancer 3: 821-831, 2003.

3. Crewe HK, Notley LM, Wunsch RM, Lennard MS and Gillam EM: Metabolism of tamoxifen by recombinant human cytochrome P450 enzymes: Formation of the 4-hydroxy, 4'-hydroxy and N-desmethyl metabolites and isomerization of trans-4-hydroxytamoxifen. Drug Metab Dispos 30: 869-874, 2002.

4. Guo WZ, Wang Y,Umeda E, Shiina I, Dan S and Yamori T: Search for novel anti-tumor agents from ridaifens using JFCR39, a panel of human cancer cell lines. Biol Pharm Bull 36: 1008-1016, 2013.

5. Cancer NCCf: 4.1 Endocrine therapy. In: Advanced Breast Cancer: Diagnosis and Treatment. National Collaborating Centre for Cancer (UK), Cardiff, pp17-21, 2009.

6. Bonneterre J, Buzdar A, Nabholtz JM, Robertson JF, ThürlimannB, von Euler M, Sahmoud T, Webster A, Steinberg M, Committee AW, et al; Investigators Committee Members: Anastrozole is superior to tamoxifen as first-line therapy in hormone receptor positive advanced breast carcinoma. Cancer 92: 2247-2258, 2001.

7. Patterson J, Furr B, Wakeling A and Battersby L: The biology and physiology of 'Nolvadex' (tamoxifen) in the treatment of breast cancer. Breast Cancer Res Treat 2: 363-374, 1982.

8. Bakkevold KE, Pettersen A, Arnesjø B and Espehaug B: Tamoxifen therapy in unresectable adenocarcinoma of the pancreas and the papilla of Vater. Br J Surg 77: 725-730, 1990.

9. Rosenberg L: Treatment of pancreatic cancer. Promises and problems of tamoxifen, somatostatin analogs, and gemcitabine. Int J Pancreatol 22: 81-93, 1997.

10. Couldwell WT, Hinton DR, Surnock AA, DeGiorgio CM, Weiner LP, Apuzzo ML, Masri L, Law RE and Weiss MH: Treatment of recurrent malignant gliomas with chronic oral high-dose tamoxifen. Clin Cancer Res 2: 619-622, 1996.

11. Pawar P, Ma L, Byon CH, Liu H, Ahn EY, Jhala N, Arnoletti JP, McDonald JM and Chen Y: Molecular mechanisms of tamoxifen therapy for cholangiocarcinoma: Role of calmodulin. Clin Cancer Res 15: 1288-1296, 2009.

12. Hoelting T, Duh Q-Y, Clark OH and Herfarth C: Tamoxifen antagonizes proliferation and invasion of estrogen receptornegative metastatic follicular thyroid cancer cells via protein kinase C. Cancer Lett 100: 89-93, 1996.

13. Scandlyn MJ, Stuart EC, Somers-Edgar TJ, Menzies AR and Rosengren RJ: A new role for tamoxifen in oestrogen receptornegative breast cancer when it is combined with epigallocatechin gallate. Br J Cancer 99: 1056-1063, 2008.

14. Chen HY, Yang YM, Stevens BM and Noble M: Inhibition of redox/Fyn/c-Cbl pathway function by Cdc42 controls tumour initiation capacity and tamoxifen sensitivity in basal-like breast cancer cells. EMBO Mol Med 5: 723-736, 2013.

15. Moodbidri MS and Shirsat NV: Activated JNK brings about accelerated apoptosis of Bcl-2-overexpressing C6 glioma cells on treatment with tamoxifen. J Neurochem 92: 1-9, 2005.

16. Maccarrone M, Fantini C, Ranalli M, Melino G and Agrò AF: Activation of nitric oxide synthase is involved in tamoxifeninduced apoptosis of human erythroleukemia K562 cells. FEBS Lett 434: 421-424, 1998

17. Kim JA, Kang YS, Jung MW, Lee SH and Lee YS: Involvement of $\mathrm{Ca}^{2+}$ influx in the mechanism of tamoxifen-induced apoptosis in HepG2 human hepatoblastoma cells. Cancer Lett 147: 115-123, 1999.

18. Shim J-H, Choi C-S, Lee E-C, Kim M-Y and Chun Y-J: Tamoxifen suppresses clusterin level through Akt inactivation and proteasome degradation in human prostate cancer cells. Biomol Ther (Seoul) 17: 25-31, 2009.

19. Grynkiewicz G, Poenie M and Tsien RY: A new generation of $\mathrm{Ca}^{2+}$ indicators with greatly improved fluorescence properties. J Biol Chem 260: 3440-3450, 1985.

20. Lippman M, Bolan G and Huff K: The effects of estrogens and antiestrogens on hormone-responsive human breast cancer in long-term tissue culture. Cancer Res 36: 4595-4601, 1976.

21. Li Z, Wang N, Fang J, Huang J, Tian F, Li C and Xie F: Role of PKC-ERK signaling in tamoxifen-induced apoptosis and tamoxifen resistance in human breast cancer cells. Oncol Rep 27: 1879-1886, 2012. 
22. Berridge MJ, Bootman MD and Roderick HL: Calcium signalling: Dynamics, homeostasis and remodelling. Nat Rev Mol Cell Biol 4: 517-529, 2003.

23. Thastrup O, Cullen PJ, Drøbak BK, Hanley MR and Dawson AP: Thapsigargin, a tumor promoter, discharges intracellular $\mathrm{Ca}^{2+}$ stores by specific inhibition of the endoplasmic reticulum Ca2(+)-ATPase. Proc Natl Acad Sci USA 87: 2466-2470, 1990.

24. Racay P and Lehotský J: Intracellular and molecular aspects of $\mathrm{Ca}(2+)$-mediated signal transduction in neuronal cells. Gen Physiol Biophys 15: 273-289, 1996.

25. White CD and Sacks DB: Regulation of MAP kinase signaling by calcium. In: MAP Kinase Signaling Protocols. 2nd edition. Seger R (ed). Humana Press, Totowa, NJ, pp151-165, 2010.

26. Radin DP and Patel P: Delineating the molecular mechanisms of tamoxifen's oncolytic actions in estrogen receptor-negative cancers. Eur J Pharmacol 781: 173-180, 2016.

27. Johnson N, Bentley J, Wang LZ, Newell DR, Robson CN, Shapiro GI and Curtin NJ: Pre-clinical evaluation of cyclindependent kinase 2 and 1 inhibition in anti-estrogen-sensitive and resistant breast cancer cells. Br J Cancer 102: 342-350, 2010.

28. Zhang W, Couldwell WT, Song H, Takano T, Lin JH and Nedergaard M: Tamoxifen-induced enhancement of calcium signaling in glioma and MCF-7 breast cancer cells. Cancer Res 60: 5395-5400, 2000.

29. Lu YC, Jiann BP, Chang HT, Huang JK, Chen WC, Su W and Jan CR: Effect of the anti-breast cancer drug tamoxifen on $\mathrm{Ca}(2+)$ movement in human osteosarcoma cells. Pharmacol Toxicol 91: 34-39, 2002

30. Jones PS, Parrott E and White IN: Activation of transcription by estrogen receptor alpha and beta is cell type- and promoterdependent. J Biol Chem 274: 32008-32014, 1999.
31. Jan CR, Cheng JS, Chou KJ, Wang SP, Lee KC, Tang KY, Tseng LL and Chiang HT: Dual effect of tamoxifen, an antibreast-cancer drug, on intracellular $\mathrm{Ca}(2+)$ and cytotoxicity in intact cells. Toxicol Appl Pharmacol 168: 58-63, 2000.

32. Sandmann S and Unger T: L- and T-type calcium channel blockade - the efficacy of the calcium channel antagonist mibefradil. J Clin Basic Cardiol 2: 187-201, 1999.

33. Agabiti-Rosei E, Muiesan ML, Romanelli G, Castellano M, Beschi M, Corea L and Muiesan G: Similarities and differences in the antihypertensive effect of two calcium antagonist drugs, verapamil and nifedipine. J Am Coll Cardiol 7: 916-924, 1986.

34. Cullen PJ and Lockyer PJ: Integration of calcium and Ras signalling. Nat Rev Mol Cell Biol 3: 339-348, 2002.

35. Goldkorn T and Mendelsohn J: Transforming growth factor beta modulates phosphorylation of the epidermal growth factor receptor and proliferation of A431 cells. Cell Growth Differ 3: 101-109, 1992.

36. Zhang F, Dong W, Zeng W, Zhang L, Zhang C, Qiu Y, Wang L, Yin $X$, Zhang $C$ and Liang W: Naringenin prevents TGF- $\beta 1$ secretion from breast cancer and suppresses pulmonary metastasis by inhibiting PKC activation. Breast Cancer Res 18: 38, 2016.

37. Xiao YQ, Freire-de-Lima CG, Schiemann WP, Bratton DL Vandivier RW and Henson PM: Transcriptional and translational regulation of TGF-beta production in response to apoptotic cells. J Immunol 181: 3575-3585, 2008.

38. Perry RR, Kang Y and Greaves BR: Relationship between tamoxifen-induced transforming growth factor beta 1 expression, cytostasis and apoptosis in human breast cancer cells. Br J Cancer 72: 1441-1446, 1995 